\title{
Chiral symmetry and the pion gas virial expansion
}

\author{
A. Dobado* \\ Departamento de Física Teórica, Universidad Complutense, 28040 Madrid, Spain \\ J. R. Peláez ${ }^{\dagger}$ \\ Stanford Linear Accelerator Center, Stanford University, Stanford, California 94309
}

(Received 19 June 1998; published 22 December 1998)

\begin{abstract}
In this work we study the thermodynamic properties of the pion gas starting from the realistic elastic scattering phase shifts obtained from chiral perturbation theory and using the virial expansion. In particular we study the equation of state and the behavior of the quark condensate as a function of the temperature and the density. [S0556-2821(99)07801-7]

PACS number(s): 12.39.Fe, 11.30.Rd, 21.65.+f, 51.30.+i
\end{abstract}

\section{INTRODUCTION}

One of the most important aspects of hadronic dynamics at low energies is spontaneous chiral symmetry breaking. Indeed, when considering just the two lightest quarks as massless, there is a spontaneous breaking of $\mathrm{SU}(2)_{L}$ $\times \mathrm{SU}(2)_{R}$ down to $\mathrm{SU}(2)_{L+R}$ (isospin). As a consequence, the Nambu-Goldstone bosons associated with that breaking, namely the pions, become the relevant degrees of freedom at low energies. In the last years a general formalism called chiral perturbation theory (ChPT) has been developed [1,2], which exploits the chiral symmetry constraints, providing a phenomenological description of low-energy pion dynamics, organized as an expansion in the pion external momenta and masses (the latter appear due to the small explicit chiral symmetry breaking caused by quark masses). By using ChPT it is possible to describe at low energies a large number of processes, such as pion scattering, in terms of a small set of parameters which can be fixed from experimental data.

At high temperatures it is expected that chiral symmetry will be restored at some critical temperature, $T_{c}$, typically around a few hundred $\mathrm{MeV}$. Nevertheless, below $T_{c}$ the main excitations of the hadronic medium would be long wavelength pions or, in other words, one should deal with a hot pionic gas [3]. As is well known, finite temperature effects can be included in quantum field theory (QFT) in different ways (see [4], and references therein). In particular this is the case in ChPT, where the imaginary time formalism was used to calculate the pion gas free energy as a power expansion in the temperature over the pion decay constant, $F$, up to $\mathcal{O}\left(T^{6} / F^{6}\right)$ [5]. As a matter of fact, the thermodynamics of the pion gas has also been the subject of intense work using finite-temperature QCD sum rules [6] and other alternative approaches [7]. Among other issues, some of these calculations made it possible to obtain some $T_{c}$ estimates. In this work we will consider the interacting pionic gas, but using a different method which can be applied at

\footnotetext{
*Email address: dobado@eucmax.sim.ucm.es

${ }^{\dagger}$ On leave of absence from Departamento de Física Teórica, Universidad Complutense, 28040 Madrid, Spain. Email address: pelaez@slac.stanford.edu
}

higher temperatures and including density effects as well. Those effects are well known to be relevant in the study of the behavior of hadronic matter. In particular, once they are included in the pion gas equation of state they modify the critical temperature.

The approach that we will follow here relies on the use of the relativistic virial expansion developed a long time ago [8]. Such an expansion will allow us to obtain the relevant thermal functions in terms of the $S$ matrix, so that it is not necessary to go through the technicalities of finitetemperature QFT. As a matter of fact, it is even possible to start from an $S$ matrix obtained directly from experiment, without using any QFT. The other key point in our approach is based on the unitarity of the $S$ matrix. The standard way to consider density in statistical physics is by introducing a grand canonical ensemble, whose chemical potential should be coupled to some conserved quantum number. In the case of the pion gas, the only conserved quantities are the electric charge and the baryonic number. In particular, the total number of pions is not conserved by strong interactions. However, from pion scattering data it is well known that elastic unitarity is satisfied up to energies of about $1 \mathrm{GeV}$ (where the inelastic $K \bar{K}$ channel opens up, since the four pion channel contribution is negligible at those energies). Therefore, if one is only interested in low-energy pion dynamics or low pion gas temperatures, it is sensible to assume that the pion number is approximately conserved by strong interactions. In this way it is possible to introduce the corresponding chemical potential and a grand canonical ensemble. Thus the pion density, or the number of pions per unit of volume, becomes a meaningful physical concept, at least for temperatures well below $1 \mathrm{GeV}$.

By using the approach described above we will obtain the pion gas equation of state for low densities and temperatures below $T_{c}$. Moreover, for a given density, we will estimate this critical temperature as that where the quark condensate vanishes. The plan of the paper is as follows. In Sec. II we introduce the details of the virial expansion and discuss in detail the free gas case, including the possibility of BoseEinstein condensation. In addition, the comparison with the free gas will provide a first rough estimate of the range of applicability of the virial expansion up to the second virial coefficient, that will be complemented later with other esti- 
mates in the interacting case. In Sec. III we show our results for the real pion gas equation of state, i.e., we obtain the pressure versus the density and the temperature. In this case the second virial coefficient is computed both using the phase shifts that are obtained from standard ChPT as well as those using an inverse amplitude method (IAM) fit to pion data up to about $1 \mathrm{GeV}$. In Sec. IV we obtain the quark condensate also in terms of the temperature and the density. This will make it possible to obtain an approximate phase diagram for the pion gas. Finally, in Sec. V we list the main conclusions of this work.

\section{VIRIAL EXPANSION}

\section{A. Generalities}

Let us start by considering a pion gas consisting of $g$ different pion species [for example $g=3$ for the $\mathrm{SU}(2)_{L}$ $\times \mathrm{SU}(2)_{R}$ case]. The pressure virial expansion can be written as $[8]$

$$
P=T\left(\frac{M_{\pi} T}{2 \pi}\right)^{3 / 2} \sum_{k=1}^{\infty} B_{k}(T) e^{\beta\left(\mu-M_{\pi}\right) k}=\frac{T}{\lambda^{3}} \sum_{k=1}^{\infty} B_{k}(T) \xi^{k},
$$

where, as usual, $\beta=1 / T, M_{\pi}$ is the pion mass, $\lambda$ $=\left(2 \pi / M_{\pi} T\right)^{1 / 2}$ is the thermal de Broglie wavelength and, according to our previous discussion, $\mu$ is the chemical potential associated with the total number of pions. Note that the expansion parameter is the fugacity $\xi=\exp \left[\left(\mu-M_{\pi}\right) / T\right]$. As is well known, the limit $\xi \ll 1$ corresponds to the lowdensity regime where the virial expansion is expected to work. The density $n$ of the pion gas, i.e., the number $n$ of pions per unit of volume is given by

$$
n=\left(\frac{\partial P}{\partial \mu}\right)_{V, T} .
$$

The equation of state of the system can be obtained by replacing the chemical potential in Eq. (1) by its expression in terms of temperature and density that we can obtain from Eq. (2).

\section{B. The free-pion gas}

In order to illustrate the above formalism we will consider first the free-pion gas. This will be interesting for different reasons: first it is the simplest case and, as far as it can be treated exactly, it will provide a very nice test for the virial expansion. In particular, that will allow us to obtain a first rough estimate of the range of temperatures and densities at which it can be safely applied up to the second virial coefficient. Second, due to the Weinberg low-energy theorems, pions are weakly interacting at low energies and thus the free pion gas can be a reasonable approximation to the real pion gas in such regime. Finally, let us remember that a free boson gas can suffer a Bose-Einstein condensation at very lowtemperatures. Apart from the interest that this phase has by itself, we will see that it basically determines the applicability region of the virial expansion.
Thus, the free-pion gas pressure can be written as

$$
P=-\frac{g T}{2 \pi^{2}} \int_{0}^{\infty} d p p^{2} \log \left[1-e^{\beta[\mu-E(p)]}\right],
$$

where $E(p)=\sqrt{p 2+M_{\pi}^{2}}$ is the energy of a relativistic pion in terms of the pion momentum, $p$. The above equation can be expanded in powers of the fugacity as

$$
\begin{aligned}
P & =-\frac{g T}{2 \pi^{2}} \int_{0}^{\infty} d p p^{2} \log \left[1-\xi e^{-\beta\left(E(p)-M_{\pi}\right)}\right] \\
& =\frac{g T}{2 \pi^{2}} \int_{0}^{\infty} d p p^{2} \sum_{k=1}^{\infty} \xi^{k} \frac{e^{-k \beta\left(E(p)-M_{\pi}\right)}}{k} .
\end{aligned}
$$

Hence, comparing with Eq. (1), we get

$$
B_{k}^{(0)}(T)=\frac{g}{2 \pi^{2}}\left(\frac{M_{\pi} T}{2 \pi}\right)^{-3 / 2} \frac{1}{k} \int_{0}^{\infty} d p p^{2} e^{-k \beta\left(E(p)-M_{\pi}\right)},
$$

where the superscript (0) indicates that we are referring to the free gas case. Then the first two virial coefficients are given by

$$
\begin{aligned}
& B_{1}^{(0)}(T)=\frac{g}{\left(M_{\pi} T\right)^{3 / 2}} \sqrt{\frac{2}{\pi}} \int_{0}^{\infty} d p p^{2} e^{-\beta\left[E(p)-M_{\pi}\right]}, \\
& B_{2}^{(0)}(T)=\frac{g}{2\left(M_{\pi} T\right)^{3 / 2}} \sqrt{\frac{2}{\pi}} \int_{0}^{\infty} d p p^{2} e^{-2 \beta\left[E(p)-M_{\pi}\right]} .
\end{aligned}
$$

Notice that, in the low-temperature limit, they satisfy

$$
B_{k}^{(0)}(T) \simeq g k^{-5 / 2}+O\left(\frac{T}{M_{\pi}}\right) .
$$

By using Eqs. (2) and (4) we can obtain the density

$$
n=\frac{g}{2 \pi^{2}} \int_{0}^{\infty} d p p^{2} \frac{1}{e^{-\beta[\mu-E(p)]}-1} .
$$

Now we can study the free-pion gas, both using the exact results, or the (second order) virial expansion and then compare the results. In that way we expect to get a crude estimate of the applicability range of the virial expansion.

First of all, it is intuitively clear that we need $\xi \ll 1$. Therefore, we cannot use the virial expansion at $\mu=M_{\pi}$, which is precisely when Bose-Einstein condensation occurs. Let us briefly study this regime.

\section{Bose-Einstein condensation}

In Eq. (3) the ground-state contribution was not taken into account, although it is well known that it plays an essential role at very low temperatures. Indeed, Eq. (3) should be rewritten as 

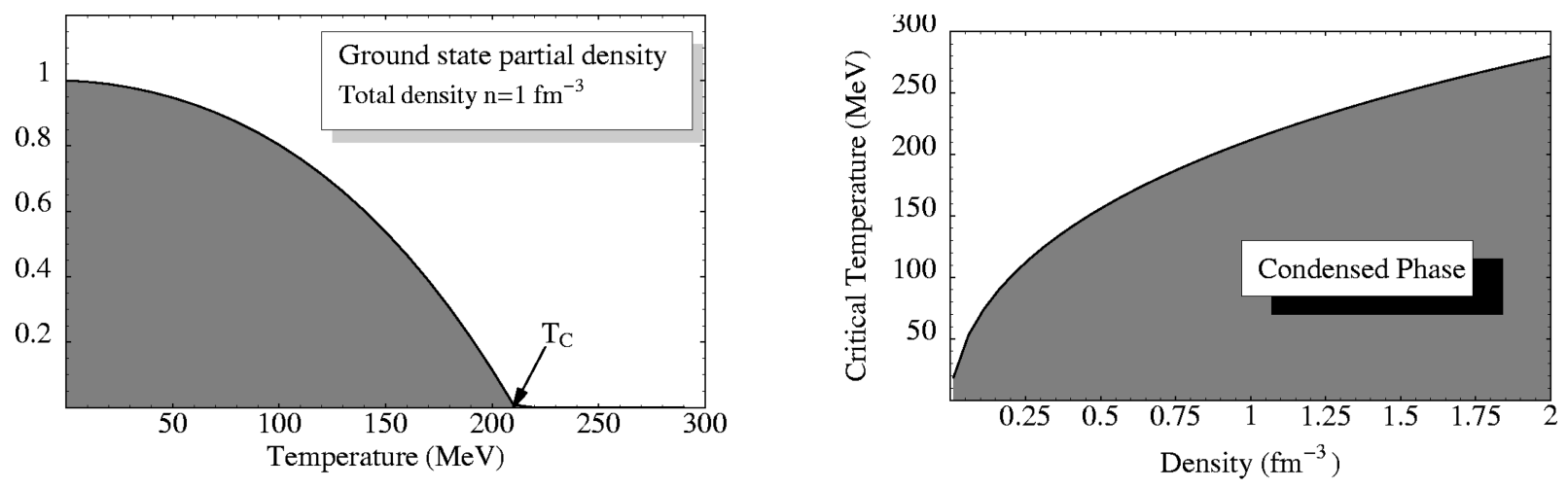

FIG. 1. Bose-Einstein condensation in the free-pion gas: (a) Evolution of the ground-state partial density $\left(n_{0} / n\right)$ with the temperature for a total density of $n=1$ pion per cubic fermi. (b) Phase diagram. We plot the critical temperature as a function of the density.

$$
\begin{aligned}
P= & -\frac{g T}{2 \pi^{2}} \int_{0}^{\infty} d p p^{2} \log \left[1-e^{\beta[\mu-E(p)]}\right] \\
& -\frac{T}{V} \log \left[1-e^{\beta\left(\mu-M_{\pi}\right)}\right]
\end{aligned}
$$

and the density as

$$
n=\frac{g}{2 \pi^{2}} \int_{0}^{\infty} d p p^{2} \frac{1}{e^{-\beta[\mu-E(p)]}-1}+\frac{1}{V} \frac{1}{e^{-\beta\left(\mu-M_{\pi}\right)}-1},
$$

where the last term is the number of pions with zero momentum per unit of volume, which form the so-called BoseEinstein condensate. We thus define

$$
n_{0}=\frac{1}{V} \frac{1}{e^{-\beta\left(\mu-M_{\pi}\right)}-1} .
$$

Let us now remember that for Eqs. (9) and (10) to make sense, the chemical potential should satisfy $\mu \leqslant M_{\pi}$. As a consequence, in the thermodynamic limit, where $N, V \rightarrow \infty$ with $N / V$ constant, we find two phases: If $\mu \leqslant M_{\pi}, n_{0}=0$. But if we lower the temperature, keeping a fixed density, $\mu$ increases until $\mu=M_{\pi}$. At that point $n_{0} \neq 0$ and the groundstate density starts to grow, forming the Bose-Einstein condensate. Eventually, at $T=0$, all pions are in the ground state so that $n_{0}=n$. The critical temperature $T_{c}$, where the phase transition occurs, can be obtained numerically from Eq. (11).

In Fig. 1(a) we show the ground-state partial density, defined as $n_{0} / n$, versus the temperature. For illustrative purposes we have chosen a total density $n=1 \mathrm{fm}^{-3}$. By using the Landau theory of phase transitions [9], it has been shown that in our case they are of second order [10].

From the above discussion it is clear that $T_{c}$ depends on the density. Using Eq. (11), we have plotted in Fig. 1(b) the critical temperature versus the density. That is the BoseEinstein condensation phase diagram of the pion gas. Let us then recall that in the condensed phase, $\mu=M_{\pi}$, which means that $\xi=1$, and therefore, we cannot apply the virial expansion.

\section{Virial expansion applicability range}

In practice, we will be using the second-order virial expansion, and we would like to know where it yields an accurate result. We have just seen that, simply because $\xi=1$, there is a range of temperatures and densities where we cannot use the virial approach. But the expansion could still be poor for values of $\xi<1$, that is, $\mu<M_{\pi}$. In Fig. 2(a) we show, in the $(T, \mu)$ plane, the error in the pressure obtained with the second-order virial expansion compared with the exact calculation using Eq. (3). Notice that the error is less than $5 \%$ if we keep $\mu \leqslant 135 \mathrm{MeV}$ and temperatures higher than $10 \mathrm{MeV}$. A similar plot can be obtained for the density, but due to its exponential dependence on the chemical potential, the invalid region is practically indistinguishable from the Bose-Einstein condensed phase [compare Fig. 2(b) with Fig. 1(b)].

Of course the interactions will modify this picture, although it will be very similar at low temperatures and densities. We have also used an additional criterium to estimate where the breaking of the virial expansion occurs. Namely, if we assume that the ratio of the unknown third coefficient to the second is the same as that of the second to the first, we will consider that the expansion is broken if the third term contribution amounts to $25 \%$. Basically this is to consider the virial coefficients of the same order of magnitude, and look for a $25 \%$ error. Or even more simply, it is similar to ask for the second term of the virial expansion to be smaller that $60 \%$ of the total result. We will show the excluded regions in the figures of Sec. IV.

Let us remark that for the free gas this method overestimates the error calculated above by a factor between 2 and 4, because in that case the ratio of two consecutive virial coefficients decreases as their order increases [see Eq. (7)].

\section{THE EQUATION OF STATE}

In this section we will apply the virial expansion to the interacting pion gas. Since the second virial coefficient is the first one sensible to the interactions we have to keep, at least, up to the second term in the virial expansion. That contribution can be written in terms of the $\pi \pi \rightarrow \pi \pi$ elastic scattering phase shifts $\delta_{I J}(E)$ as [8] 

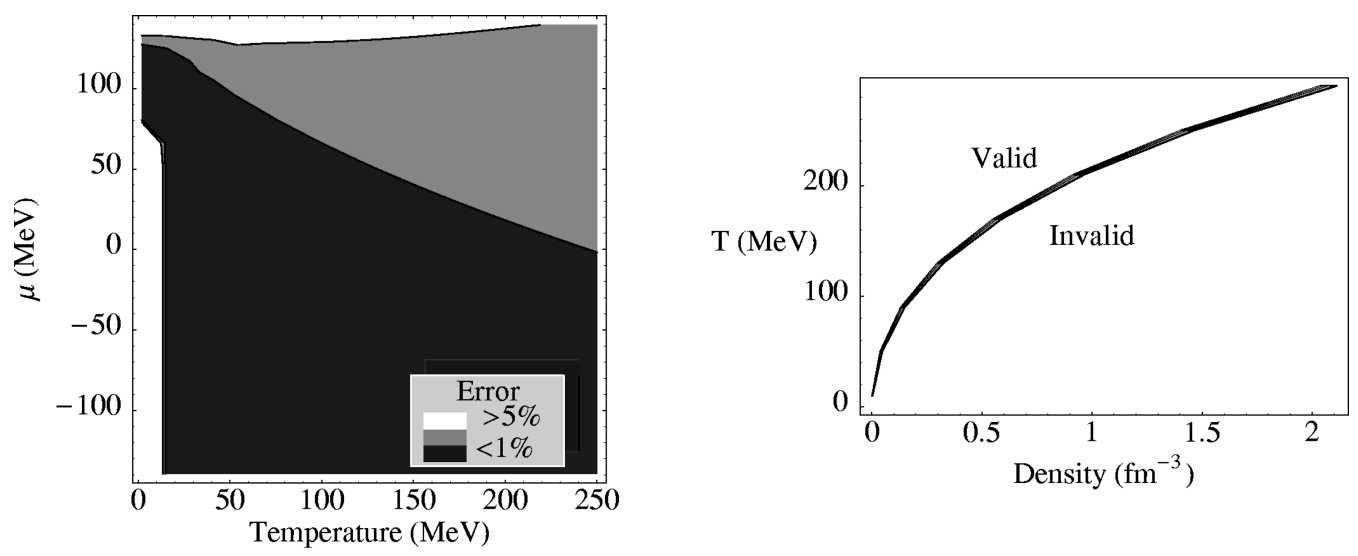

FIG. 2. Second-order virial expansion applicability range for the free gas: (a) In the $(T, \mu)$ plane, within the black area corresponds to points where the virial expansion of the pressure has less than $1 \%$ error, the grey area stands for less than $5 \%$ error, whereas white is more than 5\%. (b) Applicability region of the virial approach in the $(T, n)$ plane and the density. Note that, even demanding the error to be less than $5 \%$, in the $(T, \mu)$ plane, the invalid region is basically that where Bose-Einstein condensation occurs.

$$
\begin{aligned}
B_{2}(T)= & B_{2}^{(0)}(T)+\frac{4 e^{2 M_{\pi} / T}}{\left(2 \pi M_{\pi} T\right)^{3 / 2}} \int_{2 M_{\pi}}^{\infty} d E E^{2} K_{1}(E / T) \\
& \times\left(\sum_{I, J}(2 I+1)(2 J+1) \delta_{I J}(E)\right)
\end{aligned}
$$

where $K_{1}$ is the modified Bessel function with asymptotic behavior

$$
K_{1}(x) \simeq \sqrt{\frac{\pi}{2 x}} e^{-x}
$$

for large $x$.

It is important to notice that in Eq. (12) we could simply use the experimental phase shifts, without any reference to any underlying physical theory. In this way we can compute quantities as the pressure or the density, which is enough to obtain the equation of state. But we could not calculate their derivatives with respect to, for instance, the pion mass, which is needed to study the $\langle\bar{q} q\rangle$ condensate.

Therefore, in this work we will obtain the phase shifts with two different approaches: On the one hand, we will use ChPT to $O\left(p^{4}\right)$, with the parameters proposed in $[2,11]$ which fits the experimental data on elastic pion scattering for the $(I, J)=(0,0),(1,1),(2,0)$ channels, up to energies of the order of $0.5 \mathrm{GeV}$. The interest of using ChPT relies on the fact that it yields a systematic expansion in terms of the external momenta and masses of the pions. That will be extremely relevant in order to obtain other thermodynamic properties that may require derivatives with respect to the masses. That is indeed the case of the chiral condensate, that will be treated in the next section. On the other hand, it has been shown [12] that the ChPT fits to pion scattering can be extended to higher energies using the inverse amplitude method (IAM). Indeed, it yields remarkably good fits up to approximately $1 \mathrm{GeV}$. That could help to obtain a better estimate of the pressure, and the equation of state, although maybe not of its derivative with respect to the pion mass.
Moreover, as far as we will be interested in temperatures $T \leqslant 300 \mathrm{MeV}$, the Bessel function in Eq. (12) will suppress the $\delta(E)$ contributions at high energies. Thus we can also use the IAM phase shifts to estimate how big is the error on the pure ChPT results due to these higher energy contributions. However, note that, as far as both methods reproduce pretty well the experimental data at low energies, our results concerning the equation of state of the pion gas a low temperatures can be considered as quite realistic independently of the fact that we have used ChPT or the IAM.

Thus, from the phase shifts coming from standard ChPT and from ChPT complemented with the IAM method, together with Eqs. (6) and (12) we can obtain the second virial coefficient. Using Eq. (1) we can therefore compute the value of the pressure as a function of $T$ and $\mu$. We show the results in Fig. 3. In particular, we plot the pressure dependence on the temperature for $\mu=0$, which is well within the applicability range of the virial approach (This case is interesting since it corresponds to the canonical ensemble [5].) One of the three curves is the free-pion gas and the other two the interacting case both using standard ChPT or ChPT with the IAM.

Moreover, by solving numerically Eq. (2) we can obtain $\mu$ in terms of the density $n$ and $T$. Thus we can finally com-

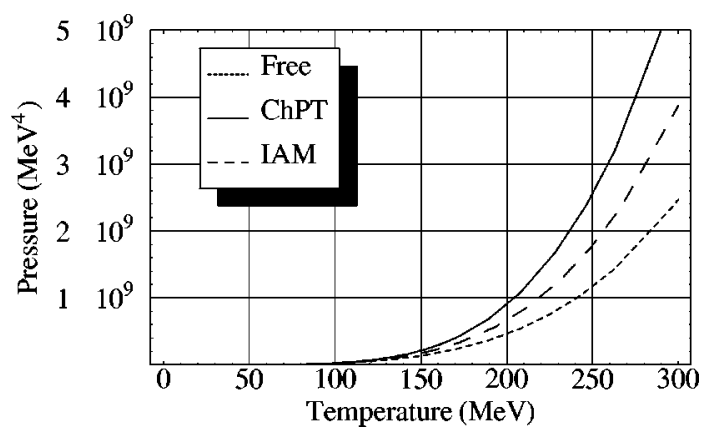

FIG. 3. The pressure of an interacting pion gas as a function of the temperature. We plot the curves both for the phase shifts obtained from pure ChPT as well as with the IAM, together with that of the free pion gas, as a reference. 

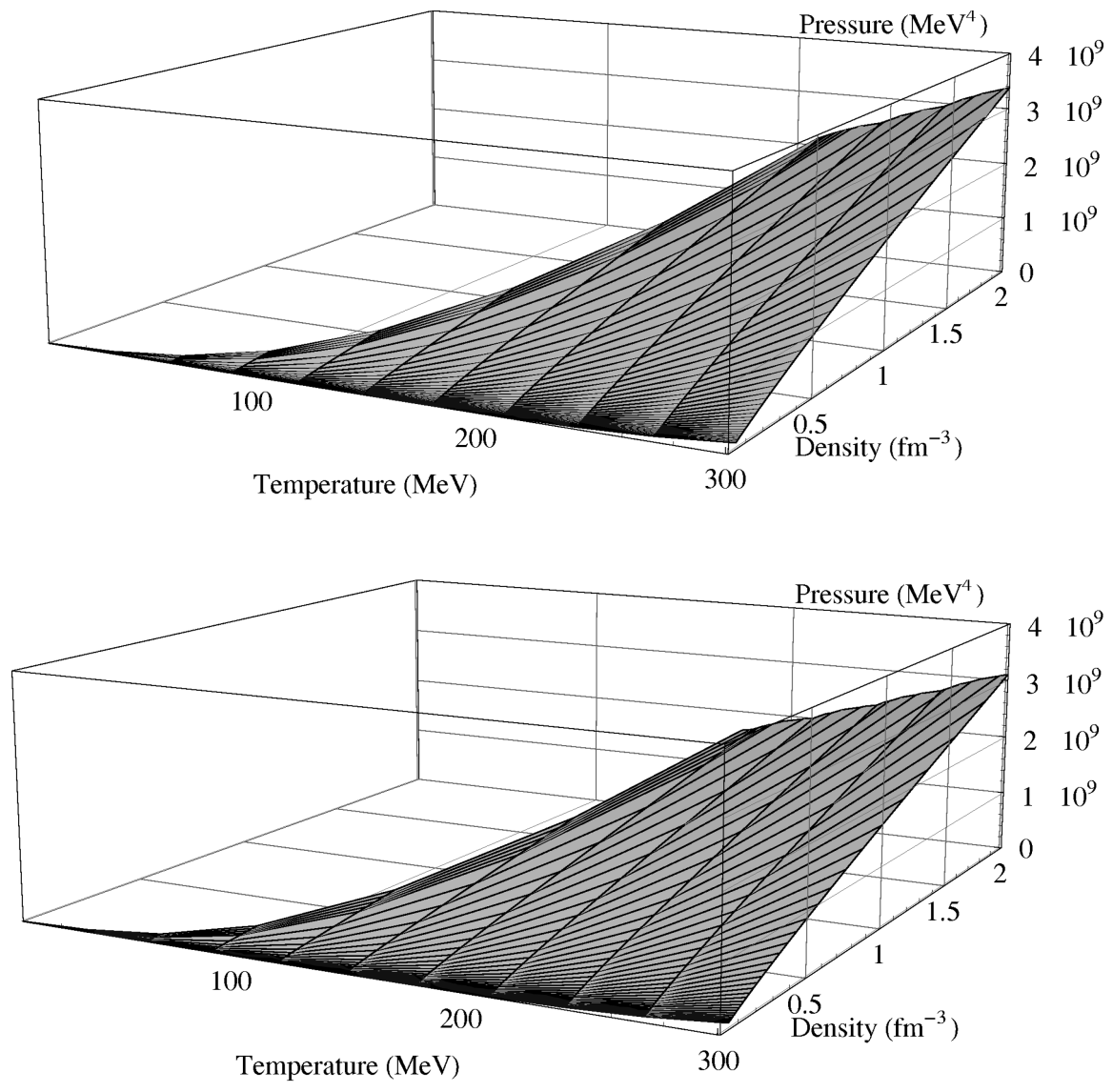

FIG. 4. Equation of state for an interacting pion gas. The blank area is out of the applicability reach of the virial approximation. We plot the pressure as a function of temperature and density: (a) using standard ChPT phase shifts. (b) complementing ChPT with the IAM. pute the pressure as a function of the temperature and the density, $P=P(T, n)$, i.e., we obtain the equation of state for a pion gas with realistic interactions. In Figs. 4(a) and 4(b) we show a three-dimensional plot of the equation-of-state surface, for standard ChPT and the IAM, respectively. As we have already discussed in the previous section, there are parts of the $(T, n)$ space that we cannot explore within the virial approach and have been left blank. Out of that area, we can trust the virial expansion. Nevertheless we expect to find some differences depending on whether we complement ChPT with the IAM or not, which should become bigger at higher temperatures. Nevertheless, it can be noticed that the numerical difference between Figs. 4(a) and 4(b), is of the order of $5 \%$ at $T=300 \mathrm{MeV}$ and $n=2 \mathrm{fm}^{-3}$. As we are going to be interested in temperatures below $300 \mathrm{MeV}$, it seems that standard ChPT yields an equation of state with sufficient accuracy.

Let us remark that we are strictly using a SU(2) model and therefore we do not have other light particles, like kaons or etas, present. In a realistic gas, the effect of these other particles should also be taken into account, although it is suppressed by Boltzmann factors. This fact makes possible to estimate the contributions of these massive states [5] in a free gas approximation. The authors of Ref. [5] concluded that the dilute gas approximation, which is indeed needed for the virial expansion, is meaningful up to $150 \mathrm{MeV}$, although it "rapidly deteriorates" at higher temperatures. Therefore, our results above these temperatures should be interpreted cautiously, and the actual numbers should not be taken too literally. Still they can yield some information on the quali- tative behavior at different temperatures and densities.

Let us also remark that it is possible to apply the virial formalism to a gas with different species. The phase shifts can also be obtained from a generalization of the IAM [13], which fits the existing data up to $1.2 \mathrm{GeV}$ and provides predictions when data are not available. However, although the present approach can be generalized to include such extensions, they are beyond our present scope.

Once we have delimited the applicability constraints of standard ChPT with the virial expansion, we will use it in the next section to study the chiral transition and the quark condensate.

\section{THE QUARK CONDENSATE}

One of the topics of hadronic physics which has not completely been settled concerns the chiral phase transition. At some critical temperature it is expected that the spontaneously broken chiral symmetry is restored giving rise to a new phase. The nature of that phase is not known but it has been argued that the transition itself is of second order for the $\mathrm{SU}(2)_{L} \times \mathrm{SU}(2)_{R}$ case [14], at least in the chiral limit, or very soft first order, i.e., with a very small latent heat [15].

However, since the broken phase occurs at low temperatures, it should be mostly made out of pions. Hence, a better understanding of the pion gas may be a useful approach to study the chiral transition. Indeed, the broken phase is characterized by a nonvanishing order parameter, which is usually identified with the quark condensate $\langle\bar{q} q\rangle_{T}$. Following [5], it can be written as 


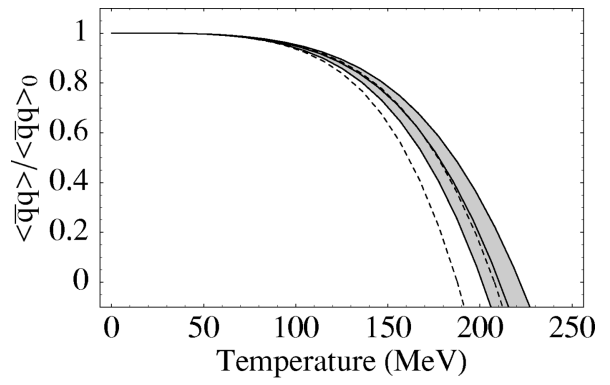

FIG. 5. Evolution of the chiral condensate with the temperature at $\mu=0$. The shaded area covers the uncertainties in the different parameters, that have been explained in the text. The area between dashed lines is the result when we add contributions from heavier particles in the free gas approximation.

$$
\frac{\langle\bar{q} q\rangle_{T}}{\langle\bar{q} q\rangle_{0}}=\left(1+\frac{c}{2 M_{\pi} F^{2}} \frac{\partial P}{\partial M_{\pi}}\right),
$$

where $F$ is basically the pion decay constant without $O\left(p^{4}\right)$ corrections, $m$ is the $u$ and $d$ averaged mass, and

$$
c=-\frac{F^{2}}{\langle\bar{q} q\rangle_{0}} \frac{\partial M_{\pi}^{2}}{\partial m} .
$$

For our calculations we have taken the numerical values considered in [5], namely, $c=0.90 \pm 0.05$ and $F=88.3$ $\pm 1.1 \mathrm{MeV}$.

In order to compute the quark condensate, and following the arguments of previous sections, we will only use the standard ChPT phase shifts. They seem accurate enough for our purposes up to the relevant temperatures and, at the same time, they have the appropriate $M_{\pi}$ dependence, which is essential in Eq. (14).

In Fig. 5 we show the evolution of the chiral condensate when $\mu=0$, for standard ChPT. The shaded area covers the uncertainties in $F$ and $c$ as well as those due to the chiral parameters $\bar{l}_{1}=-0.62 \pm 0.94, \bar{l}_{2}=6.28 \pm 0.48$ (see [11]), $\bar{l}_{3}$ $=2.9 \pm 2.4$ and $\bar{l}_{4}=4.3 \pm 0.9$ (see [2]). We have also included in the uncertainties the effect of choosing $M_{\pi}$ as that of the charged or the neutral pions, although that effect is rather small.

Let us remember once more that the $\mu=0$ results are formally equivalent to those obtained in the canonical en- semble (see [5]). The critical temperature can be estimated as the point where the condensate vanishes, and it is around 220 $\mathrm{MeV}$. These numbers should be interpreted extremely carefully, since the temperatures are quite high and the effects of other, more massive, particles could be relevant. Indeed, we can obtain a crude estimate of these contributions if we consider those massive states in a free gas approximation, following [5]. Such an approximation is justified as long as the interactions of these particles among themselves and with pions will be suppressed by Boltzmann factors. Hence, the contribution of states with masses $M_{i}$ is given by

$$
\Delta\langle\bar{q} q\rangle=-\sum_{i} \frac{\partial \Delta P}{\partial M_{i}} \frac{\partial M_{i}}{\partial \hat{m}}
$$

and therefore

$\Delta\langle\bar{q} q\rangle=\frac{1}{2 \pi^{2}} \sum_{i} g_{i} M_{i} \frac{\partial M_{i}}{\partial \hat{m}} \int_{0}^{\infty} d p \frac{p^{2}}{\sqrt{p^{2}+M_{i}^{2}} e^{\sqrt{p^{2}+M_{i}^{2}} / T}-1}$.

Following [5] we have made $\partial M_{i} / \partial \hat{m}$ to vary between $N_{i}$, the number of valence $u$ and $d$ quarks in the state of mass $M_{i}$, and $2 N_{i}$, which seems a "fair representation" of the uncertainties in that quantity. Thus, in Fig. 5, the dashed lines show how the shaded area would be modified if we take the contributions of particles with masses up to $1.5 \mathrm{GeV}$. Note that the net effect is to decrease the critical temperature by approximately $20 \mathrm{MeV}$. Indeed the contributions to Eq. (17) all tend to decrease the critical temperature. From now on we should therefore interpret the results obtained only with pions as an overestimation of the critical temperature.

Nevertheless Fig. 5 seem to indicate a clear tendency towards chiral symmetry restoration above $200 \mathrm{MeV}$. Our curves continue down to negative values, but at that point the system should be in the unbroken phase and our formalism is no longer appropriate. The results are in a remarkably good agreement with those of [5], which were obtained from a full three-loop ChPT calculation, without using the virial expansion. Their central value, including massive states, was 190 $\mathrm{MeV}$, which is compatible with our error band, although our central value lies around $200 \mathrm{MeV}$. This fact is a nice check of the error estimates of the virial expansion that we obtained

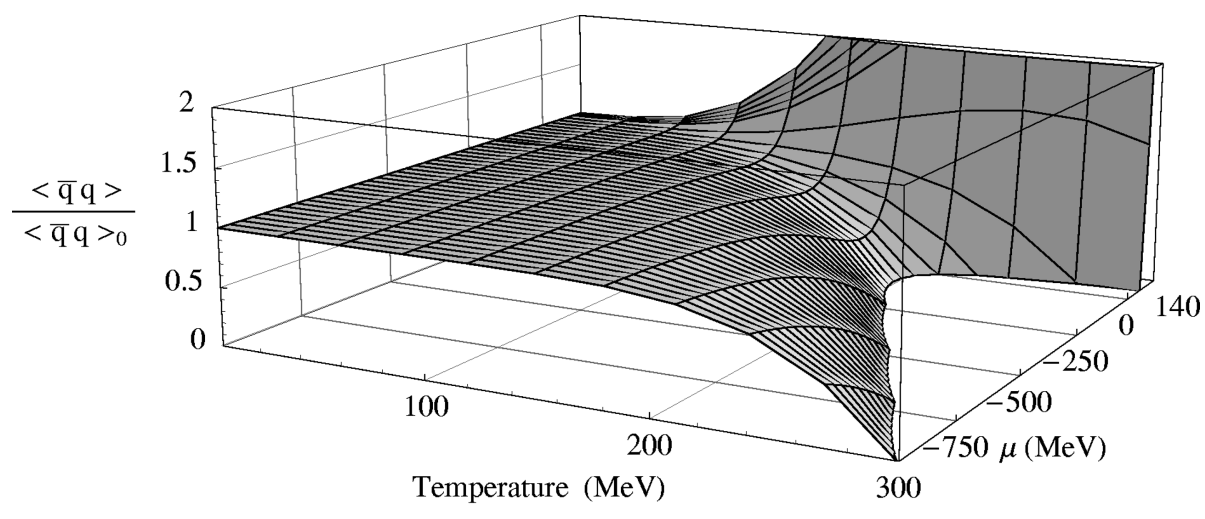

FIG. 6. The value of $\langle\bar{q} q\rangle /\langle\bar{q} q\rangle_{0}$ as a function of $\underline{T}$ and $\mu$. 


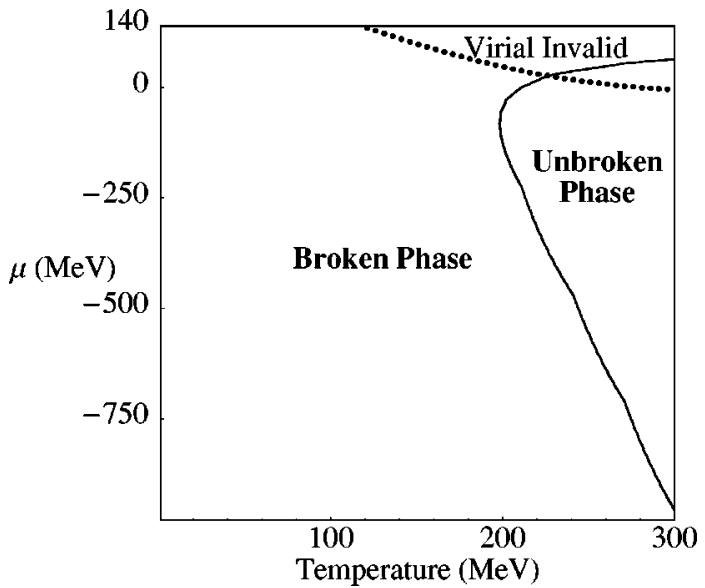

FIG. 7. Phase diagram of the chiral transition in the $(T, \mu)$ plane. The dotted line bounds the estimated applicability region of the virial approach, following Sec. II.

in Sec. II from the free gas. In addition our calculations, and provides further support for the conclusions of [5].

In order to see the effects of density on the chiral phase transition, we show in Fig. 6 the value of the chiral condensate in the $(T, \mu)$ plane. The points where $\langle\bar{q} q\rangle /\langle\bar{q} q\rangle_{0}=0$ are an estimate of the critical temperature given a fixed chemical potential. The results suggest that there are values of the chemical potential for which there is no chiral phase transition.

From now on we will also give results just for the central values of the different parameters and $M_{\pi}=139.57$.

Following the above reasoning it is therefore possible to obtain a qualitative phase diagram, which we have plotted in Fig. 7. Now we have defined the "Virial Invalid" region, where the third term of the virial expansion, when it is estimated as explained in Sec. II, is less than $25 \%$. Due to the scale, the excluded regions estimated from the free gas would give an imperceptible band close to $\mu=M_{\pi}$ and $T$ $=0$. Again, there seem to be limits, both at high and low chemical potential, where there is no phase transition (namely, $T_{C} \rightarrow \infty$ ). Moreover, for a given temperature, it seems possible, by decreasing $\mu$, to change from the broken to the unbroken phase, and back to the broken phase again.

Apparently there are several competing effects acting on the condensate. On the one hand, the temperature tends to destroy any correlation. On the other hand, the density tends to restore the broken phase if it is very high, since then the interactions of the pions can overcome the effect of the temperature (at these energies, each microscopic scattering follows the constraints of chiral symmetry breaking). But that also happens at lower densities, since the collisions are so scarce that the temperature is not able to break any existing correlation. This last effect can be easily understood since the low density limit should lead to the usual pion scattering at $T=0$. But that would be nothing but ChPT, which incorporates in its own definitions the chiral symmetry breaking.

The plots in terms of the chemical potential have the advantage that the nonapplicability region is easily defined and localized. In addition, it only occupies a tiny region of the interesting parameter space. However, the interpretation is less intuitive.

Therefore, we show in Fig. 8 the values of the chiral condensate as a function of the temperature and the density. Note that now we are using the virial expansion both for the condensate itself (since it is obtained from the pressure) and the density. Therefore, there are parts of the parameter space where we cannot apply the virial approach and have been intentionally left blank. From those results, we can extract once more the phase diagram, this time in the $(T, n)$ plane. We have plotted it in Fig. 9. Notice that the area within the dotted line is out of reach for our virial approach, due to Bose-Einstein condensation, although it seems plausible that it belongs to the broken phase. The dashed line is the bound obtained when demanding that the estimated third coefficient of the virial expansion should be less than $25 \%$. As usual, the exponential dependence of the density on $\mu$, reduces the inapplicability region to the one that lies $10-20 \mathrm{MeV}$ above Bose-Einstein condensation. Nevertheless, within the temperature and density region that we can explore, we see how the critical temperature grows extremely rapidly when we decrease the density, tending to infinity at $n \rightarrow 0$. In contrast, it is not clear whether that is also the case at high densities. From the phase diagram we can see that the critical temperature grows very softly with the density above $0.5 \mathrm{fm}^{-3}$, but we soon cross to the region where the virial approach is not applicable.

\section{CONCLUSIONS}

In this work we have studied the thermodynamic properties of a hadronic gas at low temperature and density in the

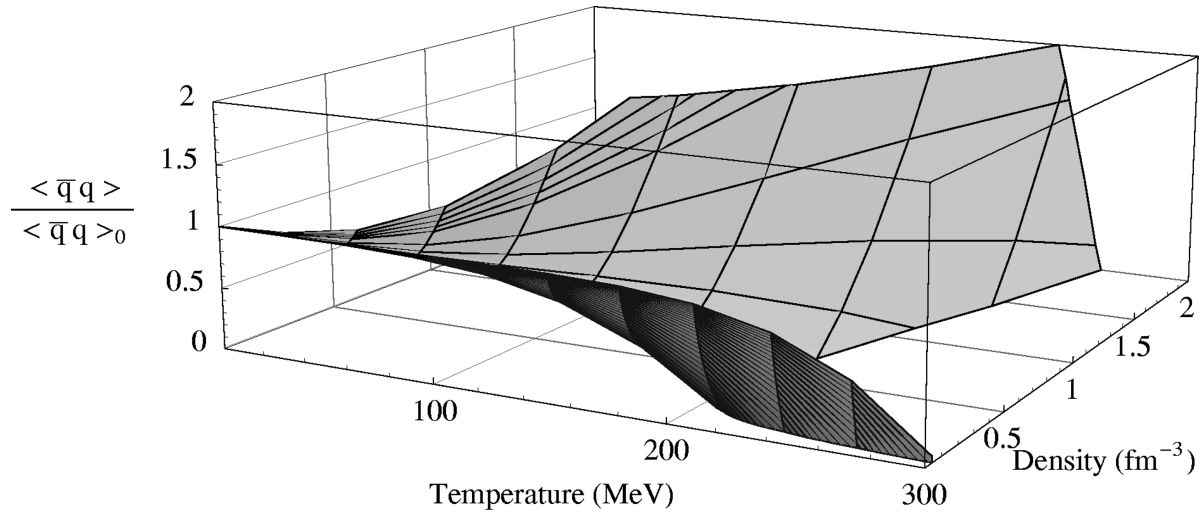

034004-7
FIG. 8. The value of $\langle\bar{q} q\rangle /\langle\bar{q} q\rangle_{0}$ as a function of the temperature and the density. 


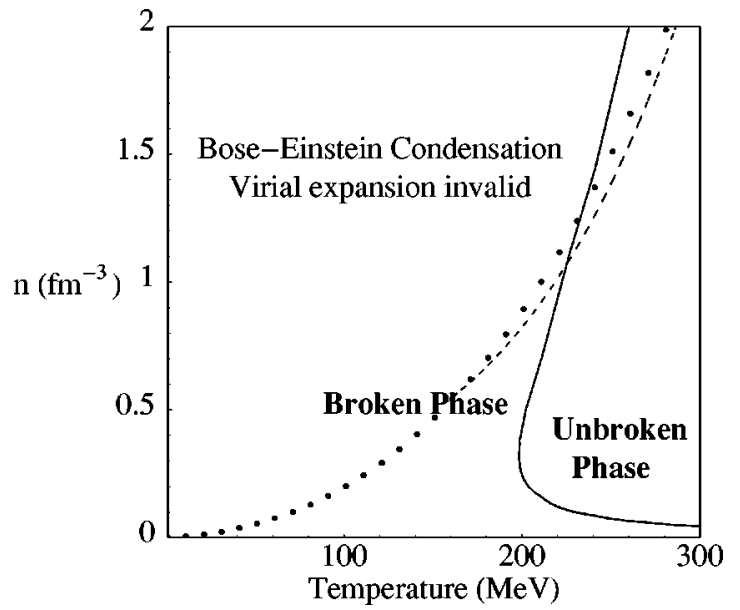

FIG. 9. Phase diagram of the chiral transition in the $(T, n)$ plane. The continuous line separates the phases with broken and unbroken chiral symmetry. The phase with Bose-Einstein condensation, where the virial expansion is not applicable, is located above the dotted line. The dashed line bounds the region where the estimated third virial term will amount to $25 \%$ of the total result, suggesting a breaking of the virial expansion.

absence of baryons. In such a case the hadronic matter can be understood as basically a gas of pions.

In order to describe the low-energy pion dynamics we have taken into account two main facts: First, that the spontaneous chiral symmetry breaking makes possible the use of ChPT to describe $\pi \pi$ elastic scattering, which fits rather well the low-energy data up to around $0.5 \mathrm{MeV}$. Using scattering amplitudes unitarized with the inverse amplitude method, which fit the data almost up to $1 \mathrm{GeV}$, we have indeed checked that, as far as $\pi \pi$ scattering is concerned, the standard ChPT approach is rather accurate in the region of interest, below the chiral phase transition.

Second, it is a matter of fact that elastic unitarity is fulfilled up to around $1 \mathrm{GeV}$. This allows us to consider the total pion number as an approximate conserved quantity at low temperatures. Hence, we can define the corresponding chemical potential and the grand canonical ensemble.

With the above considerations, we have chosen a formalism where it is possible to derive the thermodynamic functions directly from the $S$ matrix (phase shifts) by means of the virial expansion. In order to find the region where the virial expansion yields an accurate result, we have first used the free gas case, for which there are closed expressions. That has also allowed us to study the Bose-Einstein conden- sation, which, apart from the interest in itself, basically determines the region where the virial approach is not applicable. Indeed, for a given density, if we want to use the virial expansion, we have to be several $\mathrm{MeV}$ above the BoseEinstein phase transition.

Next, we have included the interactions and obtained the equation of state $P=P(T, n)$, both for the standard ChPT and unitarized scattering amplitudes. The agreement seems to be good enough to keep simply the standard description. Finally, using the interacting gas free energy we have also studied the chiral condensate dependence on the temperature and the density. Our results for zero chemical potential are in very good agreement with previous calculations using a different approach. However, by considering density effects we have now been able to obtain phase diagrams and to study the interplay of temperature, which favors the melting of the chiral condensate, with the density, which tends to enhance the chiral symmetry breaking built in the $\pi \pi$ low-energy interactions. From our phase diagrams we can learn how by diluting the hadronic gas it is possible to raise the critical temperature. At high densities, there also seems to be a very soft increase of the critical temperature, but that interpretation is more subtle due to the breaking of the virial expansion.

Of course, more detailed calculations should also consider heavier particles, like kaons or etas within the $\mathrm{SU}(3)_{L}$ $\times \mathrm{SU}(3)_{R}$ chiral scheme, as well as baryons. In this work we have only made a crude estimate of their contributions at zero chemical potential, which has allowed us to compare with previous works that do not use the virial expansion [5], checking our results and error estimates. These contributions do generally lower the critical temperature, so that our actual numbers should be understood as upper bounds on $T_{c}$, although they keep the qualitative physical features of our phase diagrams. We would finally like to stress the phenomenological nature of our work since the only ingredients are chiral symmetry, unitarity, the virial expansion, and the pion phase shifts.

\section{ACKNOWLEDGMENTS}

The research was supported by the Department of Energy under Contract No. DE-AC03-76SF00515. J.R.P. would like to thank the Theory Group at SLAC for their kind hospitality and the Spanish Ministerio de Educación y Cultura for financial support. This work has been partially supported by the Spanish CICYT under Contract No. AEN97-1693.
[1] S. Weinberg, Phys. Rev. 166, 1568 (1968); Physica A 96, 327 (1979).

[2] J. Gasser and H. Leutwyler, Ann. Phys. (N.Y.) 158, 142 (1984).

[3] E. V. Shuryak, The QCD Vacuum, Hadrons and the Superdense Matter (World Scientific, Singapore, 1988); A. V. Smilga, Phys. Rep. 291, 1 (1997).
[4] N. P. Landsman and Ch. G. van Weert, Phys. Rep. 145, 141 (1987).

[5] P. Gerber and H. Leutwyler, Nucl. Phys. B321, 387 (1989).

[6] M. Dey, V. L. Eletsky, and B. L. Ioffe, Phys. Lett. B 252, 620 (1990); V. L. Eletsky and B. L. Ioffe, Phys. Rev. D 47, 3083 (1993); 51, 2371 (1995); T. Hatsuda, Y. Koike, and S. H. Lee, Nucl. Phys. B394, 221 (1993). 
[7] G. Chanfray, M. Ericson, and J. Wambach, Phys. Lett. B 388, 673 (1996).

[8] R. Dashen, S. K. Ma, and H. J. Berstein, Phys. Rev. 187, 345 (1969).

[9] L. D. Landau and E. M. Lifshitz, Statistical Physics (Pergamon, New York, 1958).

[10] J. Kapusta, Finite Temperature Field Theory (Cambridge University Press, Cambridge, England, 1989).
[11] C. Riggenbach, J. F. Donoghue, J. Gasser, and B. Holstein, Phys. Rev. D 43, 127 (1991).

[12] A. Dobado and J. R. Peláez, Phys. Rev. D 47, 4883 (1993); 56, 3057 (1997).

[13] J. A. Oller, E. Oset, and J. R. Peláez, Phys. Rev. Lett. 80, 3452 (1998); hep-ph/9804209.

[14] F. Wilczek, Int. J. Mod. Phys. A 7, 3911 (1992).

[15] H. Leutwyler, Phys. Lett. B 284, 106 (1992). 